\title{
Foundation Settlement Prediction Based on a Novel NGM Model
}

\author{
Peng-Yu Chen and Hong-Ming Yu \\ Faculty of Engineering, China University of Geosciences, No. 388, Lumo Road, Wuhan 430074, China \\ Correspondence should be addressed to Hong-Ming Yu; andycoyl@hotmail.com
}

Received 4 December 2013; Accepted 18 February 2014; Published 23 March 2014

Academic Editor: Gradimir Milovanović

Copyright (C) 2014 P.-Y. Chen and H.-M. Yu. This is an open access article distributed under the Creative Commons Attribution License, which permits unrestricted use, distribution, and reproduction in any medium, provided the original work is properly cited.

\begin{abstract}
Prediction of foundation or subgrade settlement is very important during engineering construction. According to the fact that there are lots of settlement-time sequences with a nonhomogeneous index trend, a novel grey forecasting model called NGM $(1,1, k, c)$ model is proposed in this paper. With an optimized whitenization differential equation, the proposed NGM $(1,1, k, c)$ model has the property of white exponential law coincidence and can predict a pure nonhomogeneous index sequence precisely. We used two case studies to verify the predictive effect of $\operatorname{NGM}(1,1, k, c)$ model for settlement prediction. The results show that this model can achieve excellent prediction accuracy; thus, the model is quite suitable for simulation and prediction of approximate nonhomogeneous index sequence and has excellent application value in settlement prediction.
\end{abstract}

\section{Introduction}

Excessive settlement of foundation, subgrade, and so forth, especially differential settlement, can severely harm buildings and structures. Therefore, it is very important to observe settlement during engineering construction. Through analysis of settlement data, the development trend of current settlement is judged and the ultimate settlement is predicted so that corresponding measures can be timely taken to avoid damage to buildings and structures due to excessive settlement. At present, settlement forecasting methods based on measured data mainly include statistical prediction method, neural network, and grey theory [1-3]. In this paper, the work is focused on the application of grey forecasting model in settlement prediction.

The grey forecasting model has been widely applied in the field of geotechnical engineering, since it was proposed [4-6]. GM $(1,1)$ model, as the uppermost grey forecasting model, is the most frequently used one $[7,8]$. However, simulative sequences of GM $(1,1)$ model and various improved GM $(1,1)$ models are all homogeneous index sequences, while lots of data sequences in the field of geotechnical engineering have a nonhomogeneous index trend, such as foundation settlement-time sequences [9] and pile foundation loaddisplacement sequences [10]. In these cases, GM $(1,1)$ model is just applicable for short-term prediction of settlement rather than medium- and long-term prediction. For mediumand long-term prediction, a grey forecasting model based on nonhomogeneous index sequence should be adopted.

Many scholars have studied grey forecasting models based on nonhomogeneous index sequence [11-15]. NGM $(1,1, k)$ model is one of those models that substantially differ from other similar models. From the modeling method of $\operatorname{NGM}(1,1, k)$ model, it can be seen that there are only two parameters in its definition, while three parameters are set in other similar models. Then, does NGM $(1,1, k)$ model whose parameters are one fewer than other similar models have the same prediction performance as others? Through analysis, this paper points out that parameter setting is the fatal flaw of NGM $(1,1, k)$ model, which severely affects its application value. According to parameter settings of other similar models, this paper proposes a novel NGM model$\operatorname{NGM}(1,1, k, c)$ model.

Because of the inherent deviation existed in NGM $(1,1, k, c)$ model, this paper optimizes the parameters of the whitenization differential equation of the model to make it a better match with the grey differential equation, thus realizing prediction unbiasedness of $\operatorname{NGM}(1,1, k, c)$ model. Change in the whitenization differential equation is based on parameter reconstruction; the general solution of the whitenization 
TABLE 1: The simulative values and errors of GM $(1,1)$ model and NGM $(1,1, k)$ model.

\begin{tabular}{|c|c|c|c|c|c|}
\hline & \multirow{2}{*}{ Original value } & \multicolumn{2}{|c|}{ NGM $(1,1, k)$ model } & \multicolumn{2}{|c|}{ GM $(1,1)$ model } \\
\hline & & Simulative value $\widehat{x}^{(0)}(k)$ & Error $\varepsilon(k)$ & Simulative value $\widehat{x}^{(0)}(k)$ & Error $\varepsilon(k)$ \\
\hline$x^{(0)}(1)$ & 8.600 & 8.600 & $0.00 \%$ & 8.600 & $0.00 \%$ \\
\hline$x^{(0)}(2)$ & 9.320 & 5.501 & $40.97 \%$ & 9.211 & $1.17 \%$ \\
\hline$x^{(0)}(3)$ & 10.184 & 9.077 & $10.87 \%$ & 10.201 & $0.17 \%$ \\
\hline$x^{(0)}(4)$ & 11.221 & 11.065 & $1.39 \%$ & 11.297 & $0.68 \%$ \\
\hline$x^{(0)}(5)$ & 12.465 & 12.171 & $2.36 \%$ & 12.511 & $0.37 \%$ \\
\hline$x^{(0)}(6)$ & 13.958 & 12.786 & $8.39 \%$ & 13.855 & $0.74 \%$ \\
\hline$\varepsilon($ avg) & & & $10.66 \%$ & & $0.52 \%$ \\
\hline
\end{tabular}

differential equation is substituted into the grey differential equation to find the new parameters of the whitenization differential equation, thus establishing an optimized NGM $(1,1, k, c)$ model. Finally, the optimized NGM $(1,1, k, c)$ model is applied in settlement prediction. The results of case studies show that this model can achieve excellent prediction accuracy which is better than that of GM $(1,1)$ model and $\operatorname{NGM}(1,1, k)$ model and also superior to Asaoka model and hyperbolic model commonly used for settlement prediction, and therefore it has certain application value in settlement prediction.

\section{The Modeling Method of NGM $(1,1, k)$ Model and Its Flaw}

Definition 1. Consider

$$
x^{(0)}(k)+a z^{(1)}(k)=k b,
$$

where $z^{(1)}(k)=(1 / 2)\left(x^{(1)}(k)+x^{(1)}(k-1)\right)$, is called a grey differential equation of $\operatorname{NGM}(1,1, k)$ model (Abbreviate as $\operatorname{NGM}(1,1, k))$, which is the defining type of $\operatorname{NGM}(1,1, k)$ model. The parameter $a$ in NGM $(1,1, k)$ model is called the development coefficient and $k b$ is grey action quantity just like $b$ in $\operatorname{GM}(1,1)$ model.

The parameters of $\operatorname{NGM}(1,1, k)$ model determined by least-squares method can be written as

$$
\begin{gathered}
{\left[\begin{array}{ll}
a & b
\end{array}\right]^{T}=\left(B^{T} B\right)^{-1} B^{T} Y} \\
B=\left[\begin{array}{ccc}
-z^{(1)}(2) & 1 \\
-z^{(1)}(3) & 1 \\
\vdots & \vdots \\
-z^{(1)}(n) & 1
\end{array}\right], \quad Y=\left[\begin{array}{c}
x^{(0)}(2) \\
x^{(0)}(3) \\
\vdots \\
x^{(0)}(n)
\end{array}\right] .
\end{gathered}
$$

The equation

$$
\frac{d x^{(1)}(t)}{d t}+a x^{(1)}(t)=t b
$$

is called a whitenization differential equation of $\operatorname{NGM}(1,1, k)$ model.

The equation

$$
\begin{array}{r}
\widehat{x}^{(1)}(k)=\left(x^{(0)}(1)-\frac{b}{a}+\frac{b}{a^{2}}\right) \cdot e^{-a(k-1)}+\frac{b}{a} k-\frac{b}{a^{2}}, \\
k=1,2, \ldots, n
\end{array}
$$

is the time response equation of $\operatorname{NGM}(1,1, k)$ model.
The restored values of $x^{(0)}(k)$ can be given by

$$
\begin{aligned}
\widehat{x}^{(0)}(k) & =\widehat{x}^{(1)}(k)-\widehat{x}^{(1)}(k-1) \\
& =\left(1-e^{a}\right)\left(x^{(1)}(1)-\frac{b}{a}+\frac{b}{a^{2}}\right) e^{-a(k-1)}+\frac{b}{a}, \\
k & =2,3, \ldots, n .
\end{aligned}
$$

$\operatorname{NGM}(1,1, k)$ model is established to broaden the application scope of grey forecasting models so that this type of model can apply to simulation and prediction of approximate nonhomogeneous index sequence. To analyze its flaw, assume that $x^{(0)}(k)=A e^{\beta(k-1)}+B$ is a nonhomogeneous index sequence of raw data; then its 1-AGO sequence is

$$
x^{(1)}(k)=C e^{\beta(k-1)}+B k+D
$$

where $C=A e^{\beta} /\left(e^{\beta}-1\right)$ and $D=-A /\left(e^{\beta}-1\right)$.

Compare (4) with (6), if

$$
B=\frac{b}{a}, \quad D=-\frac{b}{a^{2}}, \quad \beta=-a,
$$

then

$$
B=D \beta=\frac{-A \beta}{e^{\beta}-1} .
$$

In the above equation, certain correlation exists among $\beta, A$, and $B$, or there are only two independent variables; $x^{(0)}(k)=A e^{\beta(k-1)}+B$ is an arbitrary function with three parameters and no direct correlation exists among $\beta, A$, and $B$. Therefore, for $\operatorname{NGM}(1,1, k)$ model, its simulative sequence cannot express an arbitrary nonhomogeneous index sequence, and its application scope is limited. To verify the flaw of $\operatorname{NGM}(1,1, k)$ model, a $\operatorname{NGM}(1,1, k)$ model is established based on nonhomogeneous index sequence $3 \times$ $1.2^{k}+5$. See Table 1 for the raw data and prediction results.

From the results in Tables 1 and 2, it can be seen that the prediction accuracy of $\operatorname{NGM}(1,1, k)$ model which is intended to be applicable for modeling of approximate nonhomogeneous index sequence is not as good as that of GM $(1,1)$ model, let alone nonhomogeneous index sequence which is selected in the case study of this paper, while GM $(1,1)$ model is only applicable for modeling of approximate homogeneous index sequence. Either simulation accuracy or 
TABLE 2: The predictive values and errors of $\operatorname{GM}(1,1)$ model and $\operatorname{NGM}(1,1, k)$ model.

\begin{tabular}{lcccc}
\hline & \multirow{2}{*}{ Original value } & \multicolumn{2}{c}{ NGM $(1,1, k)$ model } & \multicolumn{2}{c}{ GM $(1,1)$ model } \\
& & Predictive value $\widehat{x}^{(0)}(k)$ & Error $\varepsilon(k)$ & Predictive value $\widehat{x}^{(0)}(k)$ \\
\hline$x^{(0)}(7)$ & 15.750 & 13.129 & 16.642 & 15.344 \\
$x^{(0)}(8)$ & 17.899 & 13.319 & 25.591 & 16.992 \\
$x^{(0)}(9)$ & 20.479 & 13.425 & 34.448 & 18.818 \\
\hline
\end{tabular}

prediction accuracy of $\operatorname{NGM}(1,1, k)$ model is unsatisfactory or the relative error is more than $10 \%$. As to GM $(1,1)$ model, its simulation accuracy and one-step prediction accuracy are both higher, indicating that the applicability of $\operatorname{GM}(1,1)$ model is stronger than that of $\operatorname{NGM}(1,1, k)$ model.

\section{A Novel NGM Model-NGM $(1,1, k, c)$ Model and Its Optimization}

3.1. The Modeling Method of $\operatorname{NGM}(1,1, k, c)$ Model. The above theoretical analyses and case study suggest that NGM model has its own flaw; that is, its simulative sequence cannot express an arbitrary nonhomogeneous index sequence. Compare it with other similar grey forecasting models.

The whitenization differential equation of the grey model proposed by Yu and Wei [13] is $\left(d x^{(1)} / d p\right)+a x^{(1)}=b+c(p-1)$.

The whitenization differential equation of the grey model proposed by Zhang and Gu [14] is $\left(d x^{(1)} / d t\right)+a x^{(1)}=b t+c$.

The above models both have three parameters and the parameters of solutions of their whitenization differential equations are all independent from each other. Thus, these models are both applicable for simulation and prediction of approximate nonhomogeneous index sequence, which are relatively effective models. According to similar models, a parameter must be added to $\operatorname{NGM}(1,1, k)$ model.

Definition 2. The equation

$$
x^{(0)}(k)+a z^{(1)}(k)=k b+c
$$

is called a grey differential equation of $\operatorname{NGM}(1,1, k, c)$ model (abbreviated as $\operatorname{NGM}(1,1, k, c)$ ), which is the defining type of $\operatorname{NGM}(1,1, k, c)$ model. The parameter $a$ in the NGM $(1,1, k, c)$ model is called the development coefficient and $k b+c$ is grey action quantity just like $b$ in $\operatorname{GM}(1,1)$ model.

The parameters of NGM $(1,1, k, c)$ model calculated by least-square method can be written as

$$
\left[\begin{array}{lll}
a & b & c
\end{array}\right]^{T}=\left(B^{T} B\right)^{-1} B^{T} Y
$$

where

$$
B=\left[\begin{array}{ccc}
-z^{(1)}(2) & 2 & 1 \\
-z^{(1)}(3) & 3 & 1 \\
\vdots & \vdots & \vdots \\
-z^{(1)}(n) & n & 1
\end{array}\right], \quad Y=\left[\begin{array}{c}
x^{(0)}(2) \\
x^{(0)}(3) \\
\vdots \\
x^{(0)}(n)
\end{array}\right]
$$

The first-order differential equation

$$
\frac{d x^{(1)}}{d t}+a x^{(1)}=b t+c
$$

is called a whitenization differential equation of $\operatorname{NGM}(1,1$, $k, c)$ model.

The equation

$$
\widehat{x}^{(1)}(k)=\left(x^{(1)}(1)-\frac{b}{a}+\frac{b}{a^{2}}-\frac{c}{a}\right) e^{-a k}+\frac{b}{a} k-\frac{b}{a^{2}}+\frac{c}{a}
$$

is called a time response equation of $\operatorname{NGM}(1,1, k, c)$ model.

The restored values are

$$
\widehat{x}^{(0)}(k)=\left(x^{(1)}(1)-\frac{b}{a}+\frac{b}{a^{2}}-\frac{c}{a}\right)\left(1-e^{a}\right) e^{-a k}+\frac{b}{a},
$$

where $C\left(1-e^{a}\right), b / a$, and $a$ are independent from each other, satisfying the requirement. Therefore, $\operatorname{NGM}(1,1, k, c)$ model can be taken for simulation and prediction of approximate nonhomogeneous index sequence.

3.2. The Flaw of $\operatorname{NGM}(1,1, k, c)$ Model. According to the above modeling method of $\operatorname{NGM}(1,1, k, c)$ model, a problem similar to that in GM $(1,1)$ model can be found; that is, its grey differential equation and white differential equation do not match strictly, which results in inherent deviation of the model. This problem will be analyzed below.

On the interval $[k-1, k]$, the integral form of whitenization differential equation (12) is

$$
\int_{k-1}^{k} \frac{d x^{(1)}}{d t} d t+a \int_{k-1}^{k} x^{(1)} d t=\int_{k-1}^{k}(b t+c) d t
$$

then

$$
x^{(1)}(k)-x^{(1)}(k-1)+a \int_{k-1}^{k} x^{(1)} d t=\frac{(2 k-1) b}{2}+c .
$$

Comparison between (16) and grey differential equation (15) shows that they are differences in $k$ and $(2 k-1) / 2$ as well as background value $z^{(1)}(k)=(1 / 2)\left(x^{(1)}(k)+x^{(1)}(k-1)\right)$ and $\int_{k-1}^{k} x^{(1)} d t$. Therefore, the grey differential equation and the white differential equation in $\operatorname{NGM}(1,1, k, c)$ model do not match strictly.

3.3. Optimization of $\operatorname{NGM}(1,1, k, c)$ Model. An optimization method similar to that for $\operatorname{GM}(1,1)$ model, such as improved grey derivative or background value equation [16-23], would 
disobey the definition of NGM $(1,1, k, c)$ model. Reference [7] mentioned that "GM $(1,1)$ white model itself and all results derived out from the white model just establish only when they are not contradictory with the defining type, otherwise, invalid." Based on this idea, this paper improves the whitenization differential equation to make it a better match with the grey differential equation, so as to realize unbiasedness of the prediction model under the condition that the definition of $\operatorname{NGM}(1,1, k, c)$ model is obeyed and to establish an optimized NGM $(1,1, k, c)$ model (abbreviated as $\operatorname{ONGM}(1,1, k, c))$ as well.

Parameters of the whitenization differential equation are changed to match it with the grey differential equation; the whitenization differential equation with redefined parameters is written as

$$
\frac{d x^{(1)}}{d t}+m x^{(1)}=n t+p .
$$

The general solution of the above equation is

$$
x^{(1)}(k)=A e^{-m(k-1)}+\frac{n}{m} k-\frac{n}{m^{2}}+\frac{p}{m} .
$$

1-IAGO sequence of $x^{(1)}(k)$ is

$$
x^{(0)}(k)=A\left(1-e^{m}\right) e^{-m(k-1)}+\frac{n}{m} .
$$

Apparently, if (18) and (19) satisfy grey differential equation (9), the whitenization differential equation can match with the grey differential equation at this point. Substituting (18) and (19) into grey differential equation (9) gives

$$
\begin{aligned}
A e^{-m(k-1)} & {\left[\left(1-e^{m}\right)+\frac{a\left(1+e^{m}\right)}{2}\right]+\frac{a n}{m} k } \\
& +\frac{n}{m}-\frac{a n}{2 m}+a\left(\frac{p}{m}-\frac{n}{m^{2}}\right)=b k+c .
\end{aligned}
$$

To make (20) hold true, we need

$$
\begin{gathered}
\left(1-e^{m}\right)+\frac{a\left(1+e^{m}\right)}{2}=0 \\
\frac{a n}{m}=b, \quad \frac{n}{m}-\frac{a n}{2 m}+a\left(\frac{p}{m}-\frac{n}{m^{2}}\right)=c
\end{gathered}
$$

then

$$
m=\ln \frac{2+a}{2-a}, \quad n=\frac{m b}{a}, \quad p=\frac{m c}{a}-\frac{n}{a}+\frac{n}{2}+\frac{n}{m} .
$$

The above are the parameters after the whitenization differential equation of NGM $(1,1, k, c)$ model is optimized. The time response equation of the model is

$$
\begin{aligned}
\hat{x}^{(1)}(k)= & \left(x^{(1)}(1)-\frac{n}{m}+\frac{n}{m^{2}}-\frac{p}{m}\right) e^{-m(k-1)} \\
& +\frac{n}{m} k-\frac{n}{m^{2}}+\frac{p}{m} .
\end{aligned}
$$

The restored values are

$$
\widehat{x}^{(0)}(k)=\left(1-e^{a}\right)\left(x^{(1)}(1)-\frac{n}{m}+\frac{n}{m^{2}}-\frac{p}{m}\right) e^{-m(k-1)}+\frac{n}{m} .
$$

\section{Validation of White Exponential Law Coincidence}

Similar to the flaw of GM $(1,1)$ model, the whitenization differential equation and the grey differential equation does not strictly match, which results in that $\operatorname{NGM}(1,1, k, c)$ model cannot completely fit a pure nonhomogeneous index sequence.

A pure nonhomogeneous index sequence $X^{(0)}=\{d q+$ $\left.o, d q^{2}+o, d q^{3}+o, d q^{4}+o, d q^{5}+o\right\}$ is used to verify the unbiasedness of prediction of $\operatorname{ONGM}(1,1, k, c)$ model.

Accumulating the generation of $X^{(0)}$, we get

$$
\begin{gathered}
X^{(1)}=\left\{d q+o, d\left(q+q^{2}\right)+2 o, d\left(q+q^{2}+q^{3}\right)+3 o,\right. \\
\left.d \sum_{i=1}^{4} q^{i}+4 o, d \sum_{i=1}^{5} q^{i}+5 o\right\} .
\end{gathered}
$$

Substituting all data values into the calculation formula (10) for parameters gives

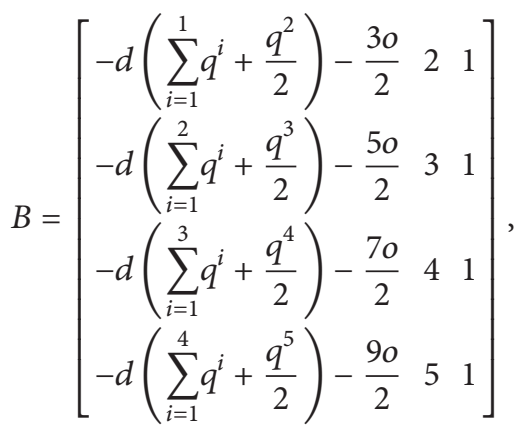

$$
\begin{aligned}
& Y=\left[\begin{array}{l}
d q^{2}+o \\
d q^{3}+o \\
d q^{4}+o \\
d q^{5}+o
\end{array}\right]^{T}
\end{aligned}
$$

$$
\begin{aligned}
{\left[\begin{array}{lll}
a & b & c
\end{array}\right]^{T} } & =\left(B^{T} B\right)^{-1} B^{T} Y \\
& =\left[\begin{array}{lll}
\frac{2-2 q}{1+q} & \frac{(2-2 q) o}{1+q} & \frac{2 q(d+o)}{1+q}
\end{array}\right]
\end{aligned}
$$

Now we can obtain the parameters of whitenization differential equation (23) as follows:

$$
\begin{gathered}
m=\ln \frac{2+a}{2-a}=\ln \frac{2+(2-2 q) /(1+q)}{2-(2-2 q) /(1+q)}=\ln \frac{1}{q}, \\
n=\frac{m b}{a}=\frac{(2-2 q) o /(1+q)}{(2-2 q) /(1+q)} \ln \frac{1}{q}=o \ln \frac{1}{q}, \\
p=\frac{q(d+o)}{1-q} \ln \frac{1}{q}-\frac{o \ln (1 / q)}{(2-2 q) /(1+q)}+\frac{o \ln (1 / q)}{2}+o .
\end{gathered}
$$


TABLE 3: The observed values and simulative values of the above forecasting models.

\begin{tabular}{ccccccccc}
\hline \multicolumn{2}{c}{} & & \multicolumn{5}{c}{ Predictive value (cm) } \\
Number Time (day) & Observed value $(\mathrm{cm})$ & $\begin{array}{c}\text { ONGM }(1,1, k, c) \\
\text { model }\end{array}$ & $\begin{array}{c}\text { NGM }(1,1, k, c) \\
\text { model }\end{array}$ & $\begin{array}{c}\text { NGM }(1,1, k) \\
\text { model }\end{array}$ & $\begin{array}{c}\text { GM }(1,1) \\
\text { model }\end{array}$ & $\begin{array}{c}\text { Asaoka } \\
\text { model }\end{array}$ & $\begin{array}{c}\text { Hyperbolic } \\
\text { model }\end{array}$ \\
\hline 1 & 10 & 23.36 & 23.36 & 23.36 & 23.36 & 23.36 & 23.36 & 25.55 \\
2 & 20 & 43.19 & 42.18 & 29.70 & 31.97 & 60.71 & 43.44 & 44.85 \\
3 & 30 & 58.73 & 59.25 & 49.25 & 49.97 & 65.80 & 59.58 & 59.94 \\
4 & 40 & 70.87 & 72.84 & 64.82 & 64.63 & 71.31 & 72.55 & 72.06 \\
5 & 50 & 83.71 & 83.64 & 77.22 & 76.58 & 77.28 & 82.99 & 82.01 \\
6 & 60 & 92.91 & 92.23 & 87.08 & 86.30 & 83.75 & 91.38 & 90.33 \\
7 & 70 & 99.73 & 99.07 & 94.94 & 94.23 & 90.76 & 98.12 & 97.39 \\
8 & 80 & 105.08 & 104.50 & 101.20 & 100.68 & 98.36 & 103.54 & 103.45 \\
9 & 90 & 109.73 & 108.83 & 106.18 & 105.94 & 106.60 & 107.90 & 108.71 \\
10 & 100 & 112.19 & 112.27 & 110.14 & 110.22 & 115.52 & 111.41 & 113.32 \\
11 & 110 & 113.45 & 115.00 & 113.30 & 113.71 & 125.20 & 114.23 & 117.39 \\
\hline
\end{tabular}

TABLE 4: The comparison of prediction performance and predictive values of ultimate settlement among the above forecasting models.

\begin{tabular}{|c|c|c|c|c|c|c|}
\hline & $\begin{array}{c}\text { ONGM }(1,1, k, c) \\
\text { model }\end{array}$ & $\begin{array}{l}\text { NGM }(1,1, k, c) \\
\text { model }\end{array}$ & $\begin{array}{c}\text { NGM }(1,1, k) \\
\text { model }\end{array}$ & $\begin{array}{c}\text { GM }(1,1) \\
\text { model }\end{array}$ & $\begin{array}{c}\text { Asaoka } \\
\text { model }\end{array}$ & $\begin{array}{c}\text { Hyperbolic } \\
\text { model }\end{array}$ \\
\hline Mean absolute error & 0.73 & 5.07 & 5.08 & 6.77 & 1.05 & 1.87 \\
\hline Mean absolute relative error & $0.94 \%$ & $7.60 \%$ & $7.31 \%$ & $9.30 \%$ & $1.18 \%$ & $2.82 \%$ \\
\hline Mean squared error & 0.88 & 39.95 & 36.71 & 69.73 & 1.45 & 4.18 \\
\hline Predictive value of ultimate settlement $(\mathrm{cm})$ & 125.63 & 125.63 & 129.03 & $+\infty$ & 125.79 & 183.28 \\
\hline
\end{tabular}

Substitute (28) into the time response equation (23), then we can get

$$
\begin{aligned}
\hat{x}^{(1)}(k) & =\frac{q^{2} d}{q-1} e^{-(\ln (1 / q))(k-1)}+o k+\frac{q d}{q-1} \\
& =\frac{d q^{2}}{q-1} q^{k-1}+o k+\frac{q d}{q-1} .
\end{aligned}
$$

The restored values are

$$
\widehat{x}^{(0)}(k)=\frac{d q^{2}}{q-1} q^{k-2}(q-1)+o=d q^{k}+o .
$$

$\hat{x}^{(0)}(k)$ is exactly equal to $x^{(0)}(k)$, while $d, q$, and $o$ can take any value. Therefore, as long as the sequence of raw data has an approximate nonhomogeneous index trend, the optimized NGM $(1,1, k, c)$ model can be used for simulation and prediction.

\section{Application of ONGM $(1,1, k, c)$ Model in Prediction of Foundation Settlement}

5.1. Case Study 1. Close neighboring the Yangtze River, the northwest stockyard of a dock is soft subsoil; the bearing capacity of which cannot satisfy the stacking requirement of the stockyard. Gravel compaction piles with the diameter of $0.5 \mathrm{~m}$ which are arranged in a regular triangle and the drainage consolidation method of staged loading are adopted for reinforcement. The reinforced area covers the area within $10 \mathrm{~m}$ away from the stockyard [24]. 11 sets of observed values of postconstruction settlement (observation interval: $10 \mathrm{~d}$ ) at Point H16 in Area B of the stockyard are chosen as the samples, and $\Delta t=10 \mathrm{~d}$ is denoted by $\Delta k=1 ; \operatorname{ONGM}(1,1$, $k, c)$ model, $\operatorname{NGM}(1,1, k, c)$ model, $\operatorname{NGM}(1,1, k)$ model, and $\operatorname{GM}(1,1)$ model are established for prediction of the ultimate settlement, so as to provide basis for engineering construction. Meanwhile, Asaoka model (graphic method) [9] and hyperbolic model [25] commonly used for settlement prediction are established for comparison with ONGM $(1,1, k, c)$ model presented in this paper. See Table 3 for the raw data and simulation results, and see Table 4 for the prediction results of ultimate settlement.

The prediction performance of these models are compared by utilizing three indexes, namely, mean absolute error, mean absolute relative error, and mean squared error. See Table 4 for the results.

First of all, four grey forecasting models are compared. From the results in Table 4, it can be seen that the values of the indexes of GM $(1,1)$ model are the biggest, and the prediction performance of this model is the poorest; the values of indexes of $\operatorname{ONGM}(1,1, k, c)$ model are much smaller than those of other models, and the prediction performance of this model is the best; $\operatorname{NGM}(1,1, k)$ model and $\operatorname{NGM}(1,1, k, c)$ model both have inherent deviation, and their prediction performance is quite close to each other in this case. This indicates that for approximate nonhomogeneous index sequence, $\operatorname{ONGM}(1,1, k, c)$ model has a good prediction performance.

The results in Table 4 show that the predictive value of ultimate settlement of $\operatorname{GM}(1,1)$ model goes to infinity, which goes against the actual situation; the predictive value 
TABLE 5: The observed values and simulative values of the above forecasting models.

\begin{tabular}{ccccccccc}
\hline \multicolumn{2}{c}{ Number Time (day) } & Observed value $(\mathrm{cm})$ & $\begin{array}{c}\text { Predictive value (cm) } \\
\text { ONGM }(1,1, k, c)\end{array}$ & $\begin{array}{c}\text { NGM }(1,1, k, c) \\
\text { model }\end{array}$ & $\begin{array}{c}\text { NGM }(1,1, k) \\
\text { model }\end{array}$ & $\begin{array}{c}\text { GM }(1,1) \\
\text { model }\end{array}$ & $\begin{array}{c}\text { Asaoka } \\
\text { model }\end{array}$ & $\begin{array}{c}\text { Hyperbolic } \\
\text { model }\end{array}$ \\
\hline 1 & 85 & 64 & 64 & 64 & 64 & 64 & 64 & 57.47 \\
2 & 95 & 73 & 73.12 & 64.58 & 43.53 & 76.19 & 72.93 & 76.24 \\
3 & 105 & 81 & 80.69 & 73.27 & 70.51 & 80.78 & 80.63 & 85.56 \\
4 & 115 & 87 & 87.26 & 80.81 & 84.94 & 85.64 & 87.27 & 91.13 \\
5 & 125 & 93 & 92.96 & 87.36 & 92.66 & 90.80 & 92.99 & 94.83 \\
6 & 135 & 98 & 97.90 & 93.03 & 96.79 & 96.27 & 97.92 & 97.47 \\
7 & 145 & 102 & 102.18 & 97.96 & 99.00 & 102.07 & 102.17 & 99.45 \\
8 & 155 & 106 & 105.90 & 102.23 & 100.18 & 108.22 & 105.83 & 100.99 \\
\hline
\end{tabular}

TABLE 6: The comparison of prediction performance and predictive values of ultimate settlement among the above forecasting models.

\begin{tabular}{|c|c|c|c|c|c|c|}
\hline & $\begin{array}{c}\text { ONGM }(1,1, k, c) \\
\text { model }\end{array}$ & $\begin{array}{l}\text { NGM }(1,1, k, c) \\
\text { model }\end{array}$ & $\begin{array}{c}\text { NGM }(1,1, k) \\
\text { model }\end{array}$ & $\begin{array}{c}\text { GM }(1,1) \\
\text { model }\end{array}$ & $\begin{array}{c}\text { Asaoka } \\
\text { model }\end{array}$ & $\begin{array}{c}\text { Hyperbolic } \\
\text { model }\end{array}$ \\
\hline Mean absolute error & 0.139 & 5.094 & 6.548 & 1.373 & 0.142 & 3.548 \\
\hline Mean absolute relative error & $0.158 \%$ & $5.855 \%$ & $8.214 \%$ & $1.561 \%$ & $0.160 \%$ & $5.544 \%$ \\
\hline Mean squared error & 0.029 & 31.987 & 128.429 & 3.100 & 0.035 & 15.786 \\
\hline Predictive value of ultimate settlement $(\mathrm{cm})$ & 130.21 & 130.21 & 101.54 & $+\infty$ & 128.72 & 113.24 \\
\hline
\end{tabular}

of ultimate settlement of ONGM $(1,1, k, c)$ model is equal to that of $\operatorname{NGM}(1,1, k, c)$ model, with the only difference in settlement convergence trend; the predictive value of ultimate settlement of $\operatorname{NGM}(1,1, k)$ is greater than that of the former two models.

From the comparison among ONGM $(1,1, k, c)$ model, Asaoka model and hyperbolic model, it can be seen that all indexes of $\operatorname{ONGM}(1,1, k, c)$ model are smaller than those of the other two models, suggesting that ONGM $(1,1, k, c)$ model has the best prediction performance among these three models. The prediction result of Asaoka model is closer to that of $\operatorname{ONGM}(1,1, k, c)$ model, because these two models are essentially exponential models which are different only in modeling mechanism. Compared with the other two models, the prediction result of ultimate settlement from hyperbolic model is significantly greater.

5.2. Case Study 2. To construct a high-grade highway on soft silt, postconstruction settlement and embankment stability are two critical problems that must be solved. Prediction of ultimate settlement through analysis of postconstruction settlement is of great significance for the evaluation of embankment stability. The observed data of settlement of a cross-section after loading on the test section of a highway in the Pearl River Delta Region are taken as samples [26], and $\Delta t=10 \mathrm{~d}$ is denoted by $\Delta k=1$; $\operatorname{ONGM}(1,1, k, c)$ model, $\operatorname{NGM}(1,1, k, c)$ model, $\operatorname{NGM}(1,1, k)$ model, GM $(1,1)$ model, Asaoka model (graphic method), and hyperbolic model are established for prediction of the ultimate settlement. See Table 5 for the raw data and simulation results, and see Table 6 for the prediction results of ultimate settlement.

First of all, four grey forecasting models are compared. From the results in Table 6 , it can be seen that the values of the indexes of $\operatorname{NGM}(1,1, k)$ model are the biggest, and the prediction performance of this model is the poorest; the values of indexes of $\operatorname{ONGM}(1,1, k, c)$ model are much smaller than those of other models, and the prediction performance of this model is the best; the prediction performance of NGM $(1,1, k, c)$ model is slightly better than that of $\operatorname{NGM}(1,1, k)$ model; the prediction performance of $\operatorname{GM}(1,1)$ model is only second to $\operatorname{ONGM}(1,1, k, c)$ model but cannot forecast the ultimate settlement. The results in Table 6 also indicate that for approximate nonhomogeneous index sequence, ONGM $(1,1, k, c)$ model has a good prediction performance.

The results in Table 6 show that the predictive value of ultimate settlement of $\operatorname{NGM}(1,1, k, c)$ model is identical to that of $\operatorname{NGM}(1,1, k, c)$ model, which is $130.21 \mathrm{~cm}$ bigger than the last observed value of settlement which is $106 \mathrm{~cm}$; this implies that settlement is still unstable when observation is finished. The predictive value of ultimate settlement of NGM $(1,1, k)$ model is smaller than the last observed value of settlement, which goes against the actual situation.

From the comparison among $\operatorname{ONGM}(1,1, k, c)$ model, Asaoka model, and hyperbolic model, it can be seen that all indexes of $\operatorname{ONGM}(1,1, k, c)$ model are almost equal to but still smaller than those of Asaoka model and also smaller than hyperbolic model, suggesting that $\operatorname{ONGM}(1,1, k, c)$ model has the best prediction performance among these three models. Compared with the other two models, the prediction result of ultimate settlement from the hyperbolic model is significantly smaller.

\section{Conclusions}

GM $(1,1)$ model has been successfully applied in many fields, but its prediction performance is sometimes unsatisfactory since its simulative sequence is homogeneous index 
sequence. For a lot of settlement-time sequences with a nonhomogeneous index trend, GM $(1,1)$ model is not appropriate for medium- and long-term prediction of foundation settlement.

The simulative sequence of $\operatorname{NGM}(1,1, k)$ model cannot express an arbitrary nonhomogeneous index sequence and sometimes it cannot achieve a satisfactory prediction performance since this model only has two undetermined parameters. Using other similar models for reference, this paper adds a parameter to NGM model and establishes $\mathrm{NGM}(1,1$, $k, c)$ model which is a novel NGM model.

Like GM $(1,1)$ model, NGM $(1,1, k, c)$ model also has the flaw of mismatching between its grey differential equation and whitenization differential equation. Based on the idea that "GM $(1,1)$ white model itself and all results derived out from the white model just establish only when they are not contradictory with the defining type, otherwise invalid"; this paper realizes prediction unbiasedness of $\operatorname{NGM}(1,1, k, c)$ model through optimizing the parameters of the whitenization differential equation of this model.

Finally, the optimized NGM $(1,1, k, c)$ model is applied in settlement prediction. The results of case studies show that the optimized NGM $(1,1, k, c)$ model has good prediction performance which is better than that of GM $(1,1)$ model, $\operatorname{NGM}(1,1, k)$ model, and $\operatorname{NGM}(1,1, k, c)$ model and also superior to Asaoka model and hyperbolic model commonly used for settlement prediction; it has an excellent application value for approximate nonhomogeneous index sequence; thus, it is suitable for settlement prediction in geotechnical engineering.

\section{Conflict of Interests}

The authors declare that there is no conflict of interests regarding the publication of this paper.

\section{Acknowledgment}

This research was supported by the National Natural Science Foundation of China (41272377).

\section{References}

[1] G. H. Li, H. B. Liu, and X. X. Qin, "Settlement prediction of roadbed based on mixture model with exponential curve and ANN," Advanced Materials Research, vol. 663, pp. 76-79, 2013.

[2] G. Zhang, X. Xiang, and H. Tang, "Time series prediction of chimney foundation settlement by neural networks," International Journal of Geomechanics, vol. 11, no. 3, pp. 154-158, 2011.

[3] H. B. Liu, Y. M. Xiang, and Y. X. Ruan, "A multivariable grey model based on background value optimization and its application to subgrade settlement prediction," Rock and Soil Mechanics, vol. 34, no. 1, pp. 173-181, 2013.

[4] F. Xie, L. M. Zhu, and L. Z. Wang, "Modified grey system forecasting model and its application for analyzing information of landslide monitory," Chinese Journal of Rock Mechanics and Engineering, vol. 24, no. 22, pp. 4099-4105, 2005.

[5] Z. Feng, Z. Li, and Y. Li, "Application of a multi-point grey model to deformation predicting of supporting structure for deep pit," Chinese Journal of Rock Mechanics and Engineering, vol. 26, supplement 2, pp. 4319-4324, 2007.

[6] X.-P. He, X.-S. Hua, and X.-F. He, "Weighted multi-point grey model and its application to high rock slope deformation forecast," Rock and Soil Mechanics, vol. 28, no. 6, pp. 1187-1191, 2007.

[7] J. L. Deng, Grey Prediction and Decision, Press of Huazhong University of Science \& Technology, Wuhan, China, 2002.

[8] S. F. Liu and N. M. Xie, Grey System Theory and Its Application, Science Press, Beijing, China, 4th edition, 2008.

[9] A. Asaoka, "Observational procedure of settlement prediction," Soils and Foundations, vol. 18, no. 4, pp. 87-101, 1978.

[10] C. Zhao, S. Li, J. Lu, and A. Gan, "Analysis of fitting loading settlement curves of single piles by integrated exponential function," Journal of Tongji University, vol. 38, no. 4, pp. 486-492, 2010.

[11] J. Cui, Y. G. Dang, and S. F. Liu, "Novel grey forecasting model and its modeling mechanism," Control and Decision, vol. 24, no. 11, pp. 1702-1706, 2009.

[12] J. Cui, S. F. Liu, B. Zeng, and N. M. Xie, "A novel grey forecasting model and its optimization," Applied Mathematical Modelling, vol. 33, no. 4, pp. 1894-1903, 2009.

[13] D. Yu and Y. Wei, "Non-homogeneous index sequence of GM model," in Proceedings of the 16th Workshop on Grey System Theory and Its Applications, pp. 355-366, China Center of Advanced Science and Technology, 2008.

[14] W. W. Zhang and G. D. Gu, Non-Homogeneous Exponential Model of National Power Generating Capacity and Its Recursive Algorithm, Progress of Management Science and System Science, Systems Engineering Society of China, 1995.

[15] N. M. Xie, S. F. Liu, Y. J. Yang, and C. Q. Yuan, "On novel grey forecasting model based on non-homogeneous index sequence," Applied Mathematical Modelling, vol. 37, no. 7, pp. 5059-5068, 2013.

[16] Y. Zhang and Y. Wei, "The improved approach of grey derivative in GM $(1,1)$ model," Journal of Grey System, vol. 18, no. 4, pp. 375-380, 2006.

[17] Y. Zhang and Y. Wei, "An approach of GM $(1,1)$ based on optimum grey derivative," Journal of Grey System, vol. 19, no. 4, pp. 397-404, 2007.

[18] Y. N. Sun and Y. Wei, "Optimization of grey derivative in GM (1, 1) based on the discrete exponential sequence," in Proceedings of the International Symposium on Information Processing, pp. 313315, 2009.

[19] B. LI and Y. Wei, "Optimizes grey derivative of GM $(1,1)$," System Engineering Theory and Practice, vol. 29, no. 2, pp. 101-105, 2009.

[20] Y. Wang, Y. Dang, Y. Li, and S. Liu, "A new method for improving prediction precision of GM(1,1) model," Journal of Grey System, vol. 21, no. 3, pp. 301-308, 2009.

[21] L. Bao and W. Yong, "Optimized GM(1,1) grey model based on romberg algorithm," Journal of Grey System, vol. 20, no. 2, pp. 125-134, 2008.

[22] D. Q. Truong and K. K. Ahn, "An accurate signal estimator using a novel smart adaptive grey model SAGM(1,1)," Expert Systems with Applications, vol. 39, no. 9, pp. 7611-7620, 2012.

[23] Z. X. Wang, Y. G. Dang, and S. F. Liu, "An Optimal GM(1, 1) based on the discrete function with exponential law," System Engineering Theory and Practice, vol. 28, no. 2, pp. 61-67, 2008.

[24] T. Ling, X. L. Ouyang, and C. Q. Hu, "Combined prediction of settlement of composite foundation with crushed gravel piles," Journal of Lanzhou University of Technology, vol. 36, no. 5, pp. 113-116, 2010. 
[25] L. Gao, Q. Y. Zhou, X. J. Yu, and Z. H. Chen, "Analysis and model prediction of subgrade settlement for Linhai highway in China," Electronic Journal of Geotechnical Engineering, vol. 19, no. A, pp. 11-21, 2014.

[26] J. Y. Shi, C. E. Zhou, Z. X. Gao, and M. K. Lv, "Local test analysis of auto-way," in Proceedings of the 7th Conference of Soil Mechanics and Foundation Engineering, pp. 464-469, China Architecture and Building Press, 1994. 


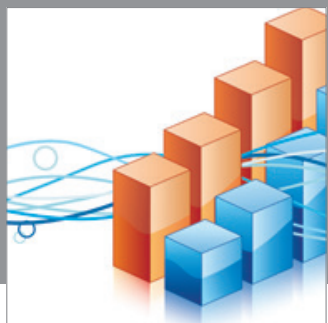

Advances in

Operations Research

mansans

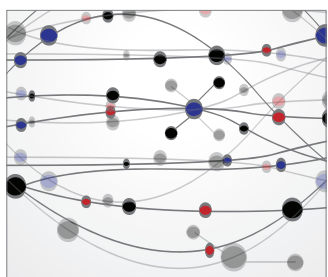

The Scientific World Journal
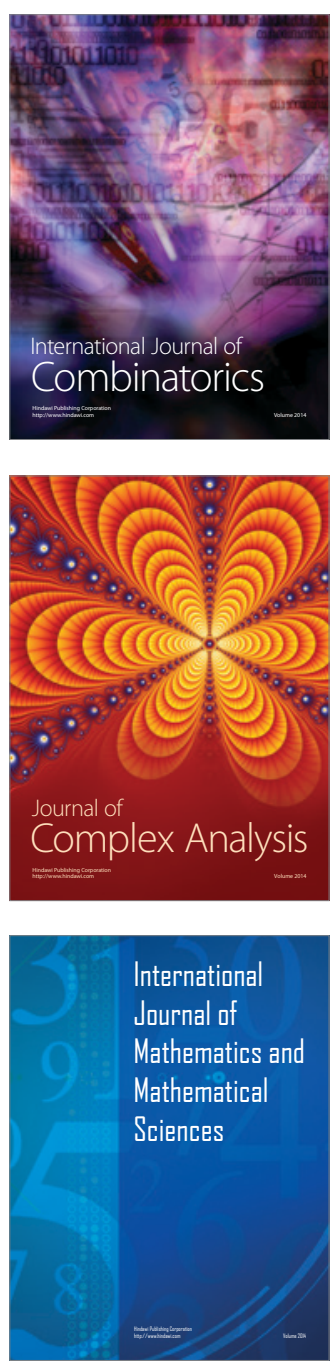
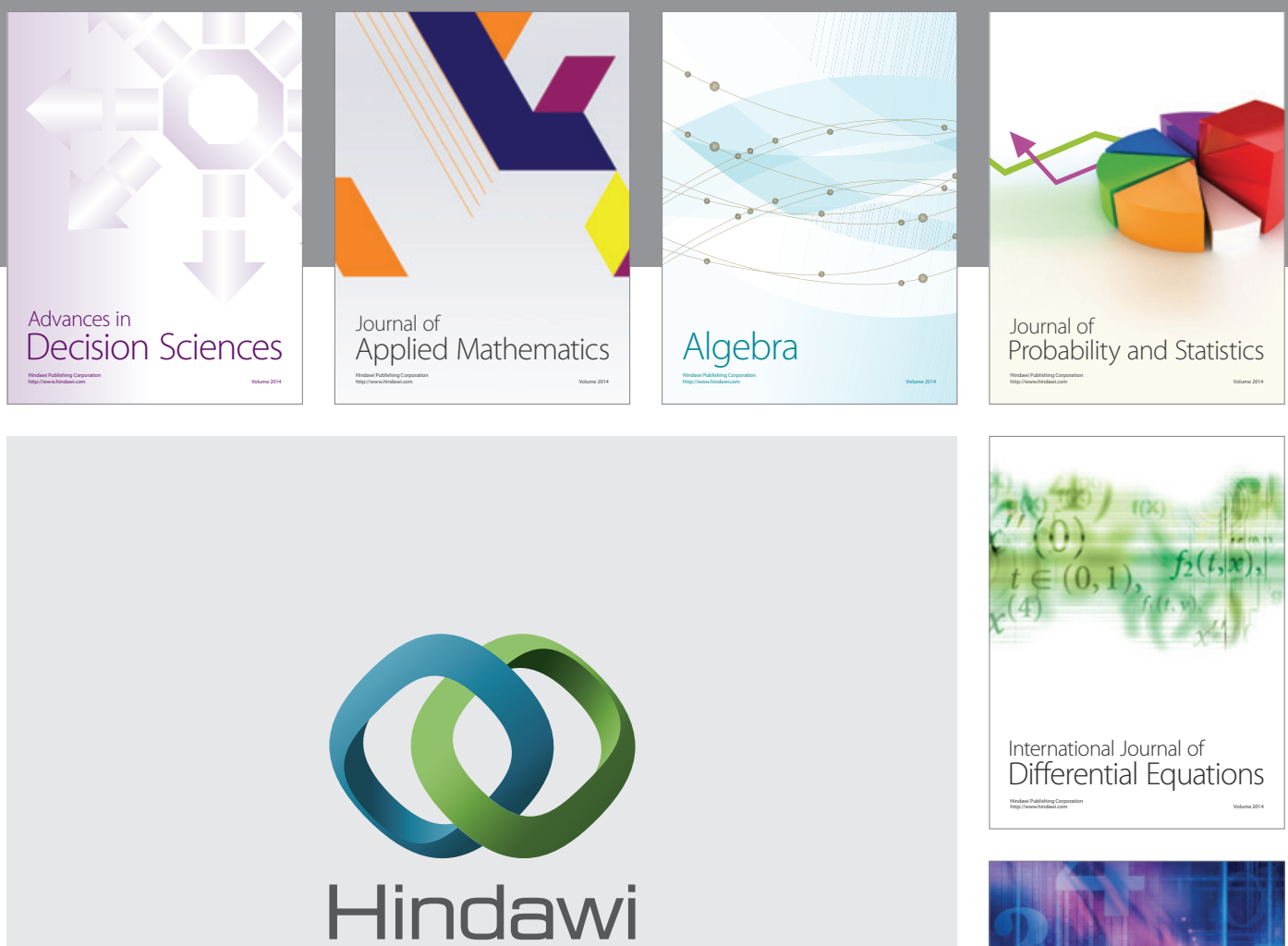

Submit your manuscripts at http://www.hindawi.com
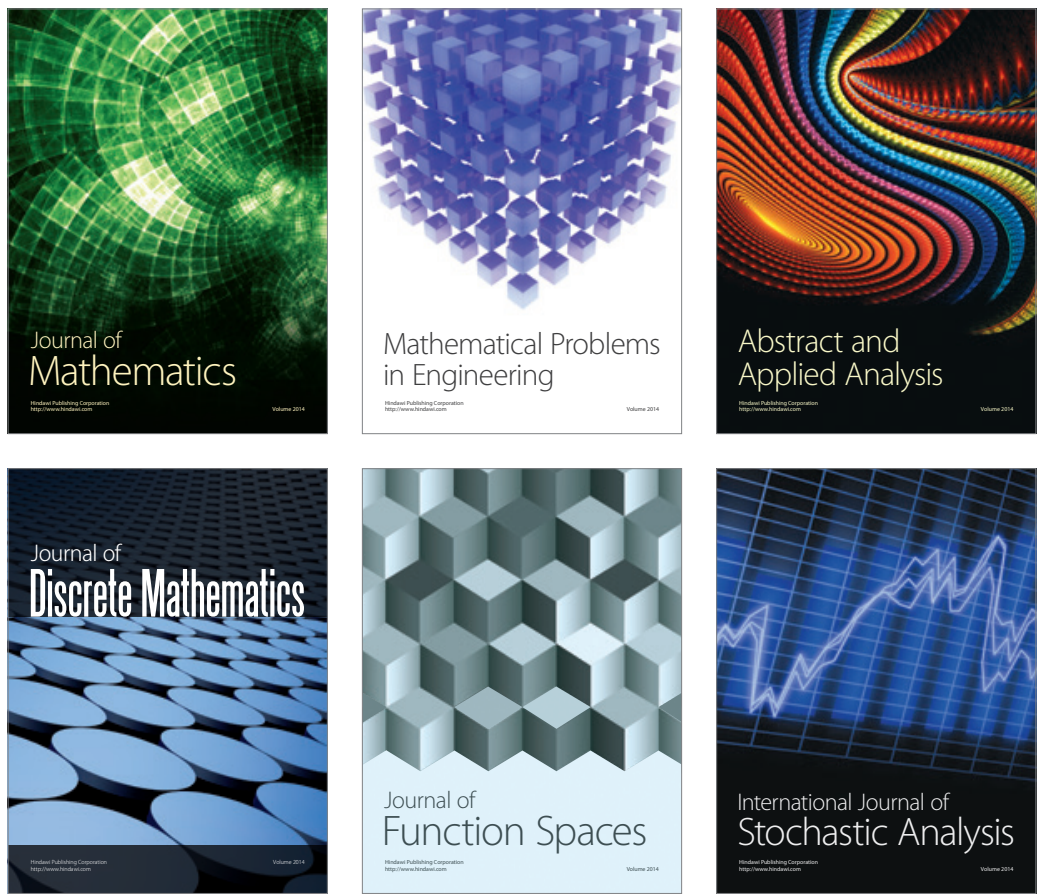

Journal of

Function Spaces

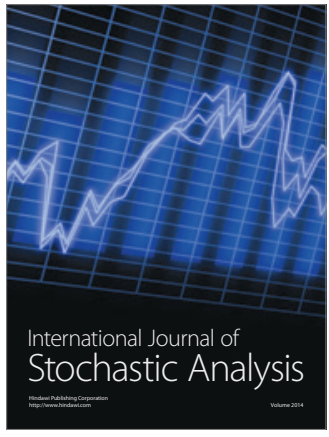

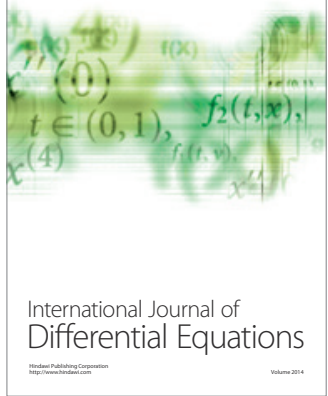
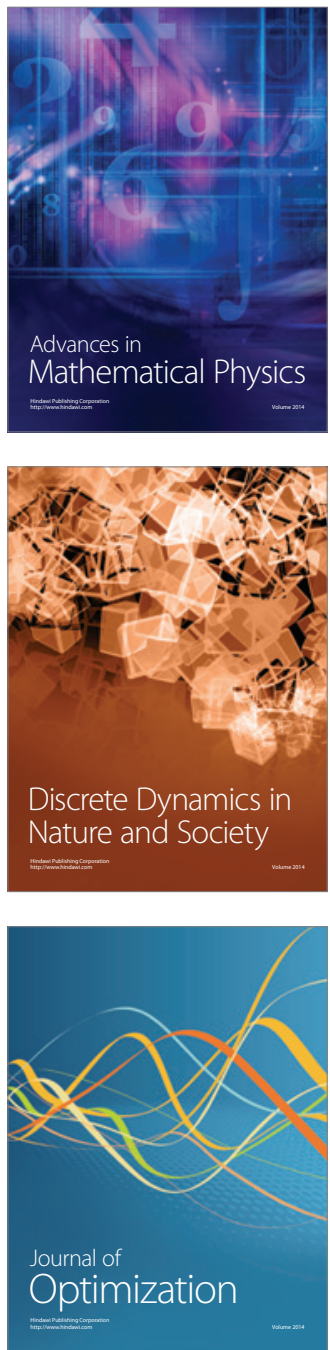